\title{
Temperature Correlations \\ for Forced-Ventilated Compartment Fires
}

\author{
K. L. FOOTE \\ Lawrence Livermore National Laboratory \\ L-442, Box 5505 \\ Livermore, California 94550, USA

\section{P. J. PAGNI} \\ Mechanical Engineering Department \\ College of Engineering \\ Berkeley, California 94720, USA \\ N. J. ALVARES \\ Lawrence Livermore National Laboratory \\ L-442, Box 5505 \\ Livermore, California 94550, USA
}

\section{ABSTRACT}

Force-ventilation compartments are a common environment for fire growth in sealed or high-rise structures. Currently, no method exists for reliably estimating the fire hazard in these enclosures. Using data from compartment fires in the forced ventilation facility at the Lawrence Livermore National Laboratory (LLNL), a simple correlation has been developed following the methods of McCaffrey, Quintiere and Harkleroad. The upper layer temperature rise above ambient, $\Delta T=T_{u}-T_{\infty}$, is given as a function of: the fire heat release rate, $\dot{0}$, the compartment mass ventilation rate, $\dot{m}$, the gas specific heat capacity, $c_{p}$, the compartment surface area $A$ and an effective heat transfer coefficient based on $\Delta T, h$. The nondimensional form of the best fit to the LLNL data is:

$$
\Delta T / T_{\infty}=0.63\left(\dot{Q} / \dot{m} c_{p} T_{\infty}\right)^{0.72}\left(h A / \dot{m} c_{p}\right)^{-0.36}
$$

This confirms the correlation suggested by McCaffrey et al. For their freeventilation data the coefficient increases to 0.77 . All the data are well fit by a coefficient of 0.7 , while the powers remain unchanged. Alternatives for, limits on, and usefulness of such correlations are discussed.

\section{INTRODUCTION}

Several methods exist for evaluating the fire hazard in traditional enclosures [1-5]. Relatively little work, however, has been done for fires in modern forced-ventilated compartments [6]. One effort to optimally describe recent forced data is presented here. A temperature correlation similar to one developed by McCaffrey, et al. [1], for naturaliy ventilated fires is sought. Given the heat release rate of the fire, the properties of the walls, the ambient temperature, the ventilation rate and room geometry, the evolution of the upper layer temperature is estimated.

Supported at LLNL by USDOE Contract No. W-7405-ENG-48 and at Berkeley by USDOC-NBS CFR Grant No. 60NANB5D0552. Assistance from B. MCCaffrey, J. Quintiere, E. Zukoski and J. Reed is gratefully acknowledged. 


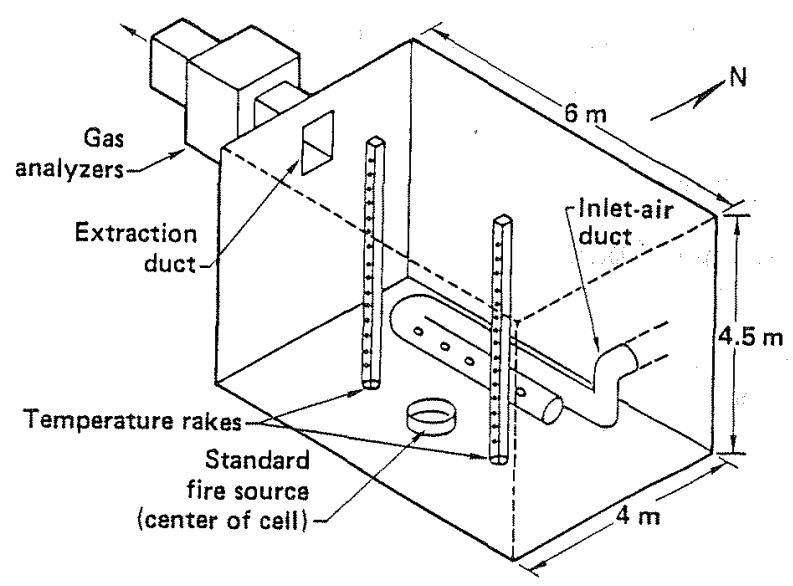

Fig. 1. Schematic of the LLNL test facility. The center of the exit opening $(0.65 \mathrm{~m} \times 0.65 \mathrm{~m})$ is $3.6 \mathrm{~m}$ above the floor. The inlet opening consisted of four wall-facing horizontal rectangles $(0.12 \mathrm{~m}$ high $\times 0.5 \mathrm{~m})$ with centerlines $0.1 \mathrm{~m}$ above the floor. All walls can be approximated as $50 \% \mathrm{Al}_{2} \mathrm{O}_{3}-50 \% \mathrm{~S}_{i} \mathrm{O}_{2}$ refractory of thickness, $\delta=0.10 \mathrm{~m}$, with conductivity, $k_{W}=0.46 \mathrm{~W} / \mathrm{m}^{\circ} \mathrm{K}$, specific heat, $c_{W}=1 \mathrm{~J} / \mathrm{g}^{\circ} \mathrm{K}$; and density, $\rho_{W}=1607 \mathrm{~kg} / \mathrm{m}^{3}$. The wall thermal penetration time is defined as $t_{p}=\delta 2 / 4 \alpha_{w}$ where $\alpha=k / \rho c$. Here $t_{p} \sim 2.5 \mathrm{hrs}$.

\section{EXPERIMENT}

The data used to develop the correlation come from a series of tests conducted during the summer of 1983 in the Lawrence Livermore National Laboratory (LLNL) fire test cell shown in Fig. 1. A detailed description of the system and its instrumentation has been published [6]. Fresh air was introduced at the floor and pulled out near the ceiling by an axial fan. A constant exit gas flow rate was controlled by a butterfly valve upstream of the fan and measured by a sharp-edged orifice. The inlet flow rate was measured using a calibrated vane anemometer. Methane gas was metered by a critical orifice into a $0.56 \mathrm{~m}$ diameter rock-filled pan in the center of the test cell floor. This burner allowed accurate control of the constant fuel flow rate and permitted a variety of heat release rates for different tests. Additional instrumentation included gas and surface temperature sensors, calorimeters, radiometers, combustion product and oxygen detectors, fuel and ventilation flow sensors, and a video camera for recording the fire shape. All measurements were recorded and reduced using an online data acquisition system.

The temperature correlation data were obtained from two thermocouple rakes positioned at $1.5 \mathrm{~m}$ on either side of the fire source. Each rake supported 15 5-mil chromel alumel thermocouples spaced $0.3 \mathrm{~m}$ apart. Figure 2 shows the evolution of the gas temperature profile in the test cell at the East rake. The upper layer is established quickly (within $150 \mathrm{~s}$ ). The upper layer gas temperature was defined as the instantaneous average of the top four thermocoup ies outside the ceiling jet. The quasi-steady approximation restricts $\dot{m}(t)$ and $\dot{Q}(t)$ to be slowly varying, i.e. no large changes ( $\geqslant 30 \%$ ) may occur on time scales fast compared with their respective characteristic times. For mass flow, 


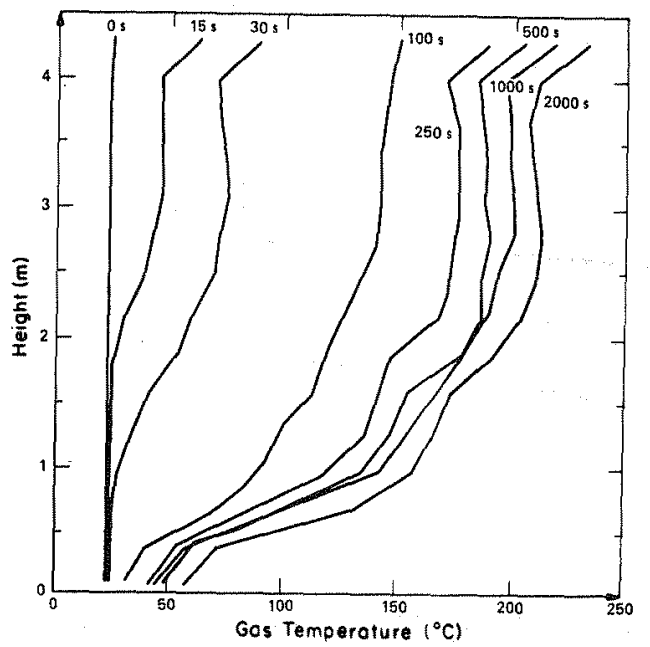

Fig. 2. Evolution of the compartment gas temperature profiles measured by the East rake shown in Fig. 1 for Test 6 (see Fig. 4). The upper layer height $(\sim 1.7 \mathrm{~m})$ and lower layer temperature may be defined as in Ref. 7 .

$t_{m}=m / \dot{m}$ or $V / \dot{V}$. Here $V \sim 10^{5} \ell$ and $\dot{V} \sim 250 \ell / \mathrm{s}$ give $t_{m} \sim 400 \mathrm{~s}$. For energy, $t_{0}=\dot{m}_{p} T / \dot{Q}$. Using the ideal gas equation of state with $R_{p}=c_{p}-c_{v}$ and $\gamma=c_{p} / c_{v}$ $=1.4$ for air gives $t_{0}=3.5 \mathrm{PV} / \dot{Q}$. Here $P \sim 1 \mathrm{~atm}, V \sim 10^{5} \ell$ and $\delta \sim 250 \mathrm{~kW}$, with $10^{-2} \ell-a t m / J$, give $t_{Q} \sim 140 \mathrm{~s}$. Since temperature is correlated here, $t_{Q}$ dominates. Species correlations will be controlled by $t_{m}$.

Figure 3 shows the histories of the inlet flow, the upper layer gas temperature and the ceiling temperature above the fire from Test 6 . Both temperatures rise quickly and then slow to their predicted approximately $[1,6]$ $\mathrm{t}^{1 / 6}$ and $\mathrm{t}^{1 / 2}$ growth rates, respectively. The rapid temperature rise coincides with the air flow out the inlet duct. This flow reversal may prove useful in suppression strategies, as dampers could be closed after much of the oxygen initially in the compartment has flowed out, thus decreasing the time to suppression.

Upper layer temperature histories from the LLNL tests are shown in Fig. 4. The approximately constant heat release and ventilation rates were chosen to be representative of possible fires in ventilation-controlled rooms with $\sim 7$ room air changes per hour.

\section{CORRELATION}

Since the compartment is open to ambient at the inlet, its pressure, $P$, is fixed near one atmosphere. The compartment volume, $V$, is fixed. So from $P V=$ $m R T$, and $E \sim m R T$ the internal energy, $E$, must be constant. Since there is no energy storage in the gas in the compartment, the energy balance is quasisteady,

$\dot{0}=\dot{m c}_{p}\left(T_{u}-T_{\infty}\right)+\dot{q}_{\text {loss }}$,

assuming gas flows in at $T_{\infty}$ and out at $T_{u}$. All the time dependence of $T_{u}(t)$ comes from the time dependence of $\dot{Q}(t), \dot{m}(t)$ and $\dot{q}(t)$. Following McCaffrey, et al. [1] the total wall heat loss is represented by a single thermal resistance, 


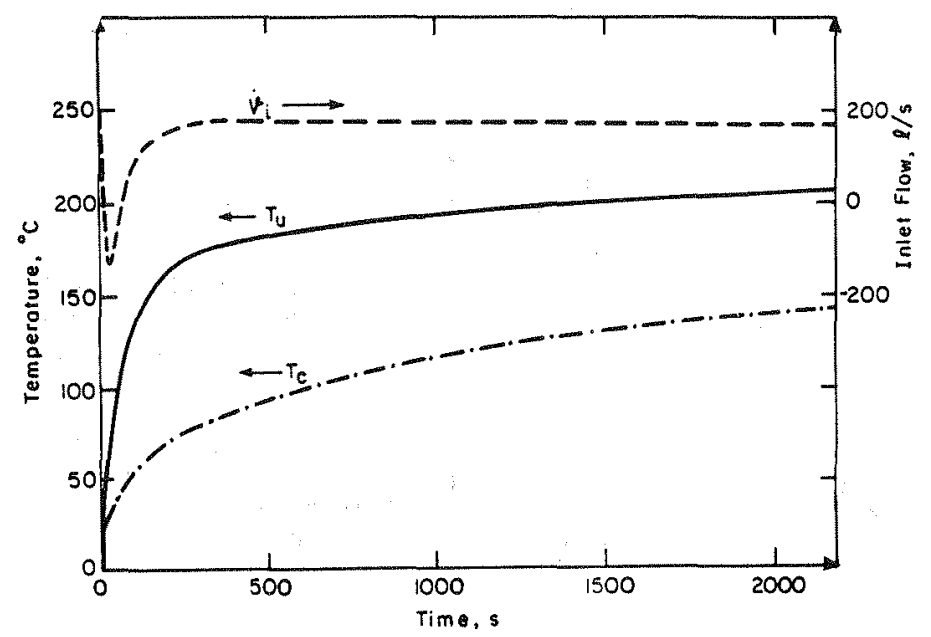

Fig. 3. Temperature and flow histories for Test 6 (see Fig. 4). $T_{C}$ is the ceiling temperature above the fire source, $\dot{V}_{i}$ is the inlet air volumetric flow rate showing a flow reversal just after ignition as the compartment dumps mass to accommodate its increased temperature.

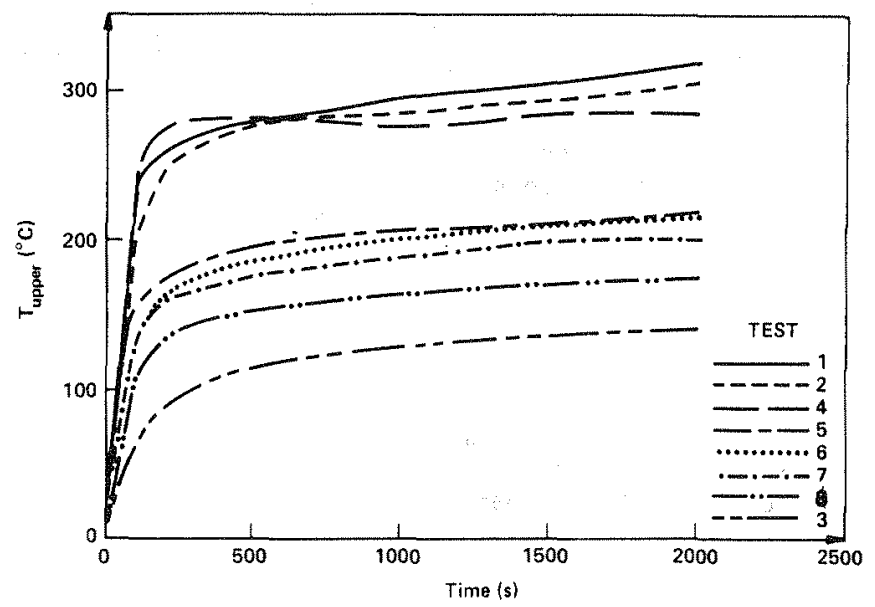

Fig. 4 Upper layer temperature histories for tests run at the following constant parameters:

$\begin{array}{lcccccccc}\text { Test } & 1 & 2 & 3 & 4 & 5 & 6 & 7 & 8 \\ T_{\infty}^{\circ}{ }^{\circ} \mathrm{K} & 307 & 303 & 282 & 298 & 295 & 298 & 300 & 284 \\ 0_{0}^{\circ} \mathrm{k} W & 490 & 465 & 150 & 400 & 230 & 250 & 250 & 180 \\ \dot{m}, \mathrm{~g} / \mathrm{s} & 300 & 160 & 190 & 220 & 110 & 175 & 325 & 180\end{array}$




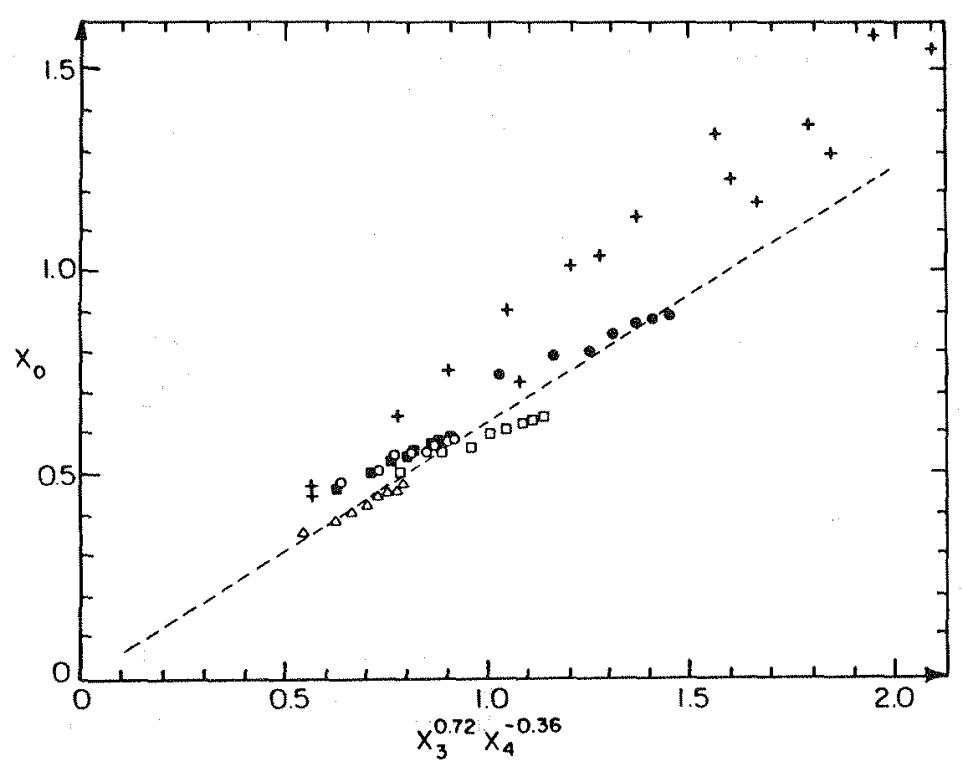

Fig. 5 Upper layer temperature data and correlation. The LLNL tests are: 1-6, $3-0,6-1,7-\square$, and $8-\Delta$. The NBS data $(+)$ are from Ref. 8. The dashed line is $\mathrm{Eq} \cdot(8)$.

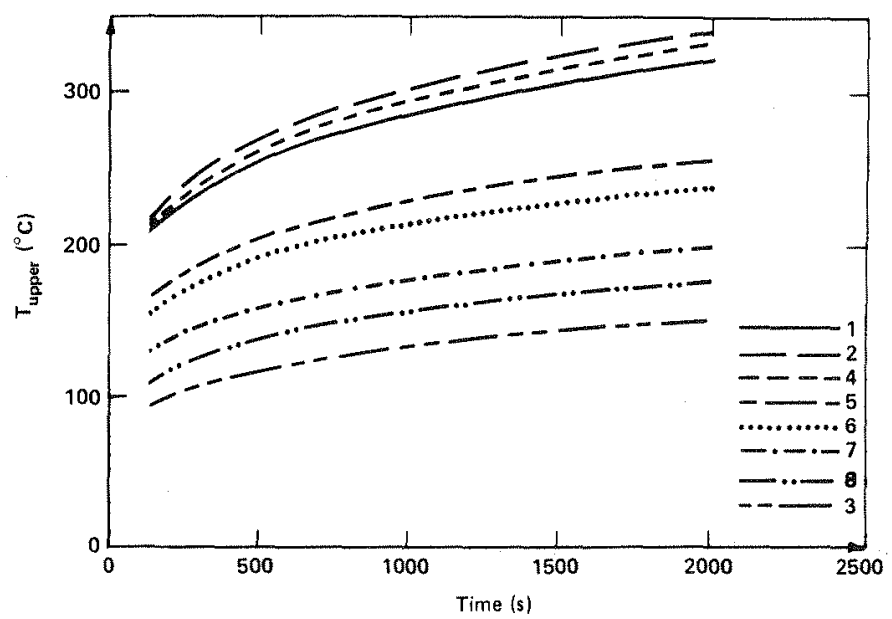

Fig. 6 Calculated upper layer temperatures using Eq. (9) with $t \geqslant 120$ s for comparison with Fig. 4. 
Many phenomena are lumped into $h$ : the radiation and convection from the hot layer and the fire to the wall, the conduction through the wall and the convection outside the compartment. The approximation is made [1] that all thermal conductances are proportional to the wall conduction, which is smallest (by $\sim 1 / 3$ ) and therefore controlling, so that

$h \sim h_{k}=\left(\rho_{w} w^{k} w^{k} / t\right)^{1 / 2}=860 t^{-1 / 2} w / m^{2}{ }^{\circ} k$,

where the last equality holds for the LLNL experiments for $t \leqslant t_{p} \sim 9000 \mathrm{~s}$. Even with the gross assumption that the overall heat transfer coefficient is proportional to $h_{k}$, there remain three time regimes with different $h_{k}(t)$. Before the thermal wave reaches the wall exterior, at $\sim t_{p} / 4$, the wall can be treated as semi-infinite and the Rayleigh problem solution gives the local heat flux,

$\dot{q}_{w}(x, t)=\left(\rho_{w} c_{w} k_{w} / \pi t\right)^{1 / 2} \Delta T \exp \left(-x /\left(4 \alpha_{w} t\right)^{1 / 2}\right)$.

At the surface, $x=0$ and $\dot{q}_{w} \sim\left(\rho w c_{w} k_{w} / t\right)^{1 / 2} \Delta T$, whence comes Eq. (3). After the finite wall thickness begins to play a role, series solutions for $\dot{q}$ are required [8]. Eventually, at $\sim 4 t_{p}$, a linear temperature profile exists in the wall and $\dot{q}=\Delta T k_{w} / \delta$. Therefore, approximate $h(t)$ as Eq. (3) for $t \leqslant t_{p}$ and as a constant, $k_{w} / \delta$, for $t>t_{p}$, and rely on the correlation to adjust for any inadequacies.

Substituting Eq. (2) into. Eq. (1) and nondimensionalizing gives

$\Delta T / T_{\infty}=\left(\dot{Q} / \dot{m} c_{p} T_{\infty}\right)\left(1+h A / \dot{m} c_{p}\right)^{-1}$ or $x_{0}=x_{3}\left(1+x_{4}\right)^{-1}$.

Equation (5) defines $x_{0}$ as the temperature rise in units of the absolute ambient temperature. $x_{3}$ is the fire strength in units of the flow sensible heat and $X_{4}$ is the ratio of the wall heat loss to the flow heat loss. $x_{1}$ and $X_{2}$ are reserved for different definitions [1],

$x_{1}=\dot{Q} /\left(A_{0} \sqrt{g H_{0}} c_{p} \rho_{\infty} T_{\infty}\right)$ and $x_{2}=h A /\left(A_{0} \sqrt{g H_{0}} c_{p} \rho_{\infty}\right)$,

for free fires where only the opening geometry (area $A_{0}$ and height $H_{0}$ ), not $\dot{m}$, is known a priori. Only the dependence, not the form of $\mathrm{Eq} .(5)$, can be relied on, so a power law fit [1] is adopted,

$x_{0}=c x_{3}^{n} x_{4}^{m}$,

as a trial expression. The corresponding correlation for free flow [1] is,

$x_{0}=1.52 x_{1}^{0.65} x_{2}^{-0.39}$.

Using this as an initial guess, an iteration process (see fig. 5) led to,

$x_{0}=0.63 x_{3}^{0.72} x_{4}^{-0.36}$. 


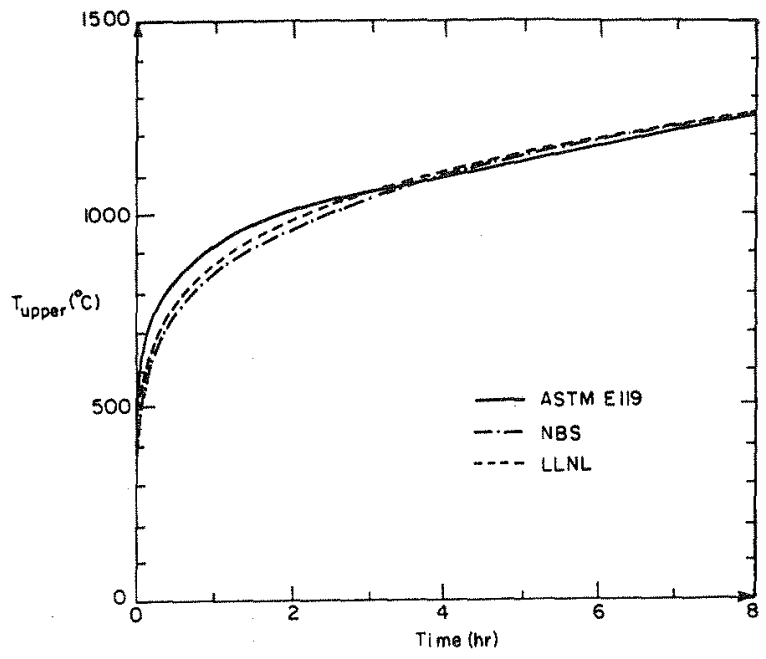

Fig. 7. Comparison of Eqs. ( 8 and 9) with the ASTM E119 Standard Time Temperature Curve showing the similar time dependencies.

The data used to obtain Eq. (9) are the well-ventilated tests for which $\dot{Q}$ was known from both mfuel and the $\mathrm{O}_{2}, \mathrm{CO}$ and $\mathrm{CO}_{2}$ exhaust mass flow rates. The dashed Tine in Fig. 5 is the fit of these points with a correlation coefficient of 0.95 . A good overall fit to both the forced and free data is obtained with $C$ in Eq. (7) as 0.70. For the NBS data $(+)$ in Fig. $5, \dot{m} \sim 0.1\left(\rho \sqrt{g H_{0}} A_{0}\right)$. The average net exponent of $\dot{m}$ in $\mathrm{Eq} .(9)$ and $\left(\rho_{\infty} \sqrt{\mathrm{gH}_{0}} \mathrm{~A}_{0}\right)$ in Eq. $(8)$ is -0.31 . So if Eqs. (6 or 8) were expressed in terms of $x_{1}$ and $X_{2}$, the coefficient $C$ would double. Comparing the 1.52 in $E q$. (8) with $(0.7)(0.1)-0.31 \approx 1.5$, shows that these correlations verify previous free results [1].

Figure 6 shows predictions from Eq. (9) for comparison with the data in Fig. 4. Test 1 is a large, well-ventilated fire with the largest $\Delta T$ observed in the LLNL data; it is well described by Eq. (9), as is the smaller Test 3 . Test 4 was initially a hot fire with little ventilation; it became underventilated and its heat release rate decreased accordingly. Tests 2 and 5 , also underventilated, were not included in the fitting process, so their agreement is encouraging. Test 7 is a well-ventilated fire that quickly reached quasi-steady conditions. Test 8 was a small isopropanol pool fire which grew more slowly than the gas fires to a steady $150 \mathrm{~kW}$.

Good agreement was also obtained for a separate series of tests with the fire source elevated $2.5 \mathrm{~m}$ above the floor. The correlation doesn't explicitly account for elevation. It can, however, be adapted to this situation by selecting $A$ as the surface area of the hot layer, i.e. the floor and ceiling areas plus only the wall area in the hot layer. This allows some heat loss below the plane of the fire and still matches the observed higher temperatures.

Comparison of Eqs. ( 8 and 9) with the American Society for Testing and Materials' standard E119 time-temperature curve is shown in Fig. 7 . Since the standard does not represent a specific compartment, the comparison had to be made by: assuming the maximum possible $X_{10 r}=10$ (see Fig. 8), calculating $X_{0}$ $=4.2$ from the ASTM curve at 8 hrs and solving for $x_{20 r 4}$. All earlier $x_{20 r 4}(t)$ $=(t / 8)-1 / 2 x_{20 r 4}(8)$. Good agreement results. 


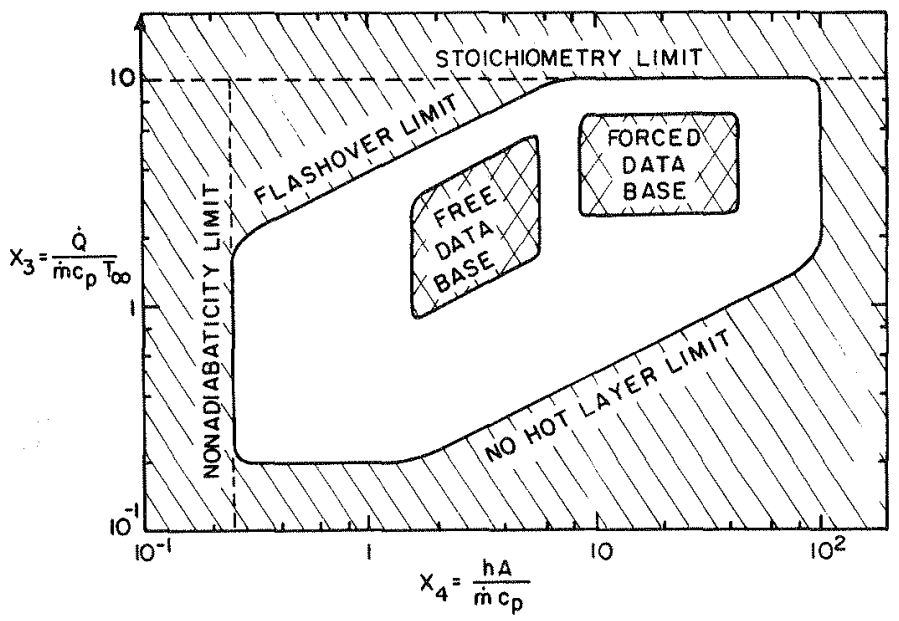

Fig. 8 Parameter space diagram showing data $(X X X)$, possible extrapolation ( ), and regions where physical constraints prohibit application of the correlations $(111)$.

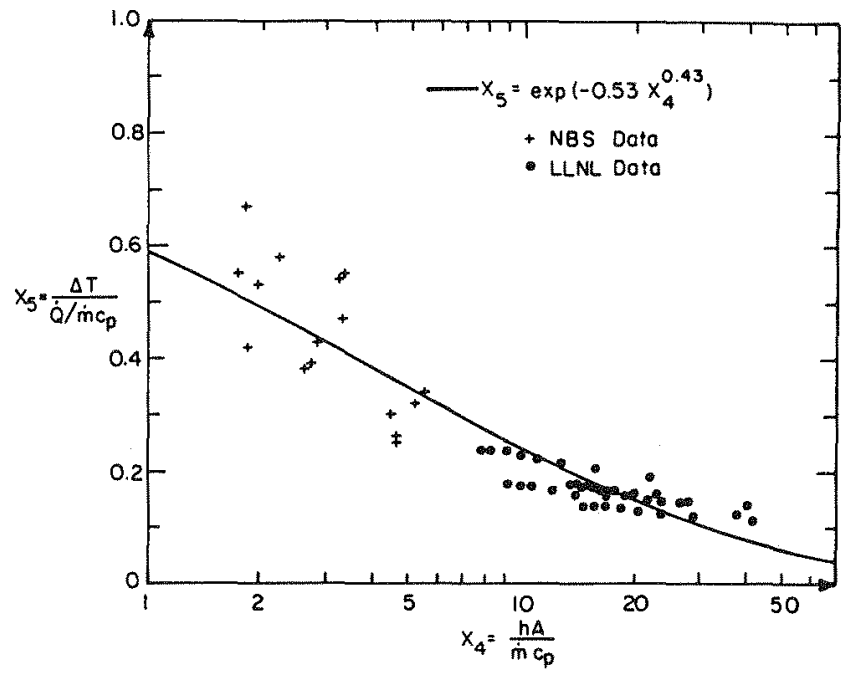

Fig. 9 The data trend in Figs. 4 and 8 and Eq. (1) suggest that the characteristic temperature is $\dot{Q} / m_{\mathrm{m}} \mathrm{c}_{\mathrm{p}}$, not $\mathrm{T}_{\text {oo. }}$. An improved correlation is shown here along with data from tests $1,3,6,7$ and $8(-)$ and Ref. $9(+)$. 
Figure 8 shows a map of regions of special interest in $x_{3}-x_{4}$ space. Test results define the inner regions on which the correlations is based. Surrounding this is the expected regime (unhatched) of valid extrapolation. Beyond this region is a space into which this correlation cannot go, for a variety of reasons, different at each boundary. $X_{3}$ cannot exceed 10 since $\dot{Q}_{\max }=\left(13 \mathrm{~kJ} / \mathrm{gO}_{2}\right)(0.23 \mathrm{g0} / \mathrm{g}$ air $) \dot{m}$, with $\mathrm{c}_{\mathrm{p}} \sim 1 \mathrm{~J} / \mathrm{gK}$ and $\mathrm{T}_{\infty} \sim 295 \mathrm{~K}$. At the other extreme an $X_{3}=0.2$ corresponds to a maximum $\Delta T$ of $\leqslant 60^{\circ} \mathrm{C}$ since if $T_{m}=$ 295 it suggests $\dot{Q} / \mathrm{mc}_{\mathrm{p}}=59^{\circ} \mathrm{C}$. This $\Delta \mathrm{T}$ is not adequate to give a stratified upper layer in most compartments [10]. The same criterion gives the diagonal no-hot-layer limit along an isotherm at $50^{\circ} \mathrm{C}$. The cutoff at $X_{4} \sim 10^{2}$ is due to the short-time limit prior to layer formation, when only detail plume analyses are permitted [12]. The nonadiabaticity limit at $x_{4} \sim 0.25$ suggests that no wall will absorb less than $20 \%$ of $\dot{0}$, since $X_{4}$ is physicaliy the ratio of the wall heat loss, hADT, to the flow heat loss. The final limit, flashover at $\Delta T=$ $500^{\circ} \mathrm{C}$, is based on the criterion suggested by McCaffrey et al. [1] and their data which show the correlation fails when $\Delta T \geqslant 500^{\circ} \mathrm{C}$. Additional data are needed to sharpen the rather fuzzy boundaries portrayed in Fig. 8 .

The general trend in Figs. 8 and 4 is that large $\dot{Q}$ leads to large $\Delta T$, i.e. large fires have hot layers and large losses. This suggests a characteristic temperature based on $\dot{Q}$ rather than $T_{\infty}$. The simple energy balance in $\mathrm{Eq}$. (1) places all $T_{\infty}$ influence into $\Delta T$. Proper nondimensionalizing of Eqs. $(1-3)$ gives the true characteristic temperature, $0 / m c_{p}$. Therefore let $X_{5} \equiv X_{0} / X_{3} \equiv$ $\Delta \mathrm{T} /\left(\dot{\mathrm{Q}} / \dot{\mathrm{m}} \mathrm{c}_{\mathrm{p}}\right)$ and seek $x_{5}\left(x_{4}\right)$. Several functional forms were considered. The data collapsed to nearly a single curve on a $\log -\log$ plot of $\ln X_{5}$ vs $X_{4}$, suggesting the form,

$x_{5}=\exp \left(-C^{\prime} x_{4}{ }^{\prime \prime}\right)$,

which gave the fit,

$x_{5}=\exp \left(-0.53 x_{4}{ }^{0.43}\right)$.

As shown in Fig. 9, Eq. (11) fits both forced and free data with a correlation coefficient of 0.91 . Also $X_{5}+1$ as $X_{4}+0$, $\dot{i} \cdot e . \Delta T=\dot{Q} / \dot{m} c_{p}$ as it should in the adiabatic limit, and $X_{5} \rightarrow 0$ as $X_{4}+\infty$, i.e. $\Delta T+0$ as expected in the limit of large losses.

Figures 8 and 9 show that, if $\Delta T \geqslant 500^{\circ} \mathrm{C}$ is a valid flashover criterion [1], flashover can not occur for $X_{4} \geqslant 10$. If $X_{4} \leqslant 10$, flashover will occur only when

$\left(\dot{0} / \dot{\mathrm{mc}} \mathrm{p}_{\mathrm{p}}\right) \exp \left(-0.53 \mathrm{x}_{4}{ }^{0.43}\right) \geqslant 500^{\circ} \mathrm{K}$.

After flashover, the upper layer no longer has a uniform temperature due to local combustion.

\section{CONCLUSIONS}

1. The fact that one fit, $x_{0}=0.7 x_{3} 0.72 x_{4}-0.36$, describes both free and forced data to within $\pm 10 \%$ and that a single simpler fit, Eq. (11) incorporates both data sets, suggests that there is no essential difference between free and forced compartment fires.

2. The distinction is that $\dot{m}$ is known in the forced case and unknown in the free. This requires the use of $X_{1}$ and $X_{2}$, in free systems and $X_{3}$ and $X_{4}$, in forced cases. 
3. This work then is a verification and extension of the temperature correlation of McCaffrey, Quintiere and Harkleroad [1]. The different range of the data in Fig. 8 is due, not to the forced $\mathrm{flow}$, but rather to the $10 \mathrm{~cm}$ thick refractory walls and larger scale of the LLNL test cell.

4. Future work should examine extension of these fits to the full range suggested by Fig. 8 and should determine if the same flashover criterion, $\Delta T$ $>500^{\circ} \mathrm{C}$, applies uniformly [12]. An improved wall heat loss term should be pursued with a more accurate $h(t)$ [13] and an area equal to the hot layer bounding surface area. The one parameter fit in Eq. (11) should be explored further.

\section{REFERENCES}

1. McCaffrey, R. J., Quintiere, J. G., and Harkleroad, M. F., "Estimating Room Temperatures and the Likelihood of Flashover Using Fire Data Correlations," Fire Technology, 17, 2, 1981 .

2. Alpert, R. L., "Turbulence Ceiling-Jet Induced by Large-Scale Fires," Combustion Science and Technology, 11, 197, 1975.

3. Quintiere, J. G., "Growth of Fire in Building Compartments," in Fire Standards and Safety, ASTM STP 614, A. F. Robertson, Ed. American Society for Testing and Materials, 131, 1977.

4. Thomas, P. H., "Fire Modeling and Fire Behavior in Rooms," Eighteen Symposium (Int.) on Combustion, The Combustion Institute, p. 503, 1981.

5. Emmons, H. W., "The Prediction of Fires in Buildings," Seventeenth Symposium (Int.) on Combustion, The Combustion Institute, p. 1101, 1978.

6. Alvares, N. J., Foote, K. L., and Pagni, P. J., "Forced Ventilation Enclosure Fires," Combustion Science and Technology, 39, 55, 1984.

7. Quintiere, J. G., Steckler, K. and Corley, D., "An Assessment of Fire Induced Flows in Compartments," Fire Science and Technology, 4, 1, 1984.

8. Carslaw, H. S. and Jaeger, J. C., Conduction of Heat in Solids, p. 102, 2nd ed., Oxford University Press, London, 1959.

9. Quintiere, J. G. and McCaffrey, B. J., "The Burning of Wood and PTastic Cribs in an Enclosure," NBSIR 80-2054, Nov. 1980.

10. Zukoski, E. E. and Kubota, T., "Two-Layer Modeling of Smoke Movement in Building Fires," Fire and Materials, 4, 19, 1980.

11. Zukoskt, E. E., "Fluid Dynamic Aspects of Room Fires," Plenary Lecture, This Symposium.

12. Thomas, P. H., Bullen, M. L., Quintiere, J. G. and McCaffrey, B. J., "Flashover and Instabilities in Fire Behavior," Combustion and Flame, 38 , $159,1980$.

13. Quintiere, J. G., McCaffrey, B. J., and Kasiwagi, T., "A Scaling Study of a Corridor Subject to a Room Fire," Combustion Science and Technology, 18, $1,1978$. 